\title{
Saint-Marcel, les Mersans
}

$\mathrm{n}^{\circ} 068498$

\section{Françoise Dumasy}

\section{(2) OpenEdition}

Journals

Édition électronique

URL : http://journals.openedition.org/adlfi/13699

ISSN : 2114-0502

Éditeur

Ministère de la culture

Référence électronique

Françoise Dumasy, « Saint-Marcel, les Mersans », ADLFI. Archéologie de la France - Informations [En ligne], Centre, mis en ligne le 24 octobre 2014, consulté le 19 avril 2019. URL : http:// journals.openedition.org/adlfi/13699

Ce document a été généré automatiquement le 19 avril 2019

(C) Ministère de la Culture et de la Communication, CNRS 


\section{Saint-Marcel, les Mersans}

$n^{\circ} 068498$

Françoise Dumasy

Lien Atlas (MCC) : http://atlas.patrimoines.culture.fr/atlas/trunk/index.php?

ap_theme=DOM_2.01.02\&ap_bbox=1.465;46.589;1.574;46.633

1 En 1997, après 8 ans de fouille sur le réseau viaire du plateau des Mersans (cf. BSR 1996 : 92), a été élaboré un nouveau programme de recherches consacré à l'étude d'une insula du centre urbain. Cette fouille doit permettre de réfléchir sur ses modalités d'occupation, en précisant la fonction de ses différents secteurs (zones publiques, zones privées) et en observant les transformations qui l'affectent au long des siècles (destructions, reconstructions, changements d'orientation, passage du public au privé ou du privé au public). C'est en nous appuyant sur des observations déjà disponibles, et en particulier sur deux séries de prospections géophysiques, l'une menée entre 1969 et 1973 par A. Hesse et A. Tabbagh, l'autre en 1996 et 1997 par A. Kermorvant, que nous avons retenu l'insula qui s'étend entre le sanctuaire et la fontaine. Les données livrées par les fouilles et par les prospections font en effet d'elle la seule insula dont on connaisse les quatre rues qui la délimitent. Par ailleurs, elle permet de saisir le tissu urbain dans sa continuité en reliant des monuments restés jusqu'ici isolés et en insérant le sanctuaire et la fontaine dans leur environnement d'origine.

2 Un relevé microtopographique de la superficie à fouiller a permis d'enregistrer un certain nombre d'anomalies du relief dont une légère dépression ovale au centre. La superposition des courbes de niveau avec les cartes établies par les prospections géophysiques a montré que la dépression correspondait à un secteur de faible résistivité, interprété à l'époque par A. Hesse comme une éventuelle place publique. Le relevé a par ailleurs fait apparaître de très légers reliefs linéaires orientés approximativement nordsud et est-ouest, que la consultation des cadastres a permis d'identifier à d'anciennes limites parcellaires. Les travaux ont commencé par un décapage à la pelleteuse des couches de terre végétale et de terre arable sur une superficie de $1500 \mathrm{~m}^{2}$. La fouille, 
menée en aire ouverte, a donc démarré sur les derniers niveaux de destruction et d'abandon, en partie perturbés par les interventions médiévales et modernes.

Les informations suggérées par les fouilles précédentes et celles qui ont été apportées par les prospections géophysiques sur le tracé des rues à proximité de l'insula ont été confirmées et précisées. La rue orientée à $16^{\circ}$ est, qui passe au nord du sanctuaire, se poursuit jusqu'à la fontaine et au-delà : la fouille en a fait apparaître la bordure sud. Toute la moitié occidentale de l'insula (murs et bases de portique) paraît fidèle à ces lignes de force. Quant à l'hypothèse d'une orientation à $240^{\circ}$ dans la moitié orientale, elle s'est révélée exacte, la fouille ayant fait apparaître une chaussée large de 6,5 m, sur laquelle s'aligne un bâtiment rectangulaire entouré de portiques, qui présente pour l'instant cinq compartiments longs de $11 \mathrm{~m}$ et larges de 3 à 3,50 $\mathrm{m}$. Reconnu sur une surface limitée lors des fouilles précédentes, il a été interprété par J. Allain et $\mathrm{R}$. Albert comme une série de boutiques, puis par A.-M. Jouquand comme un entrepôt du type horrea (cf. BSR 1993: 97-99). Nous proposons de voir dans cet édifice, situé à l'angle de deux rues importantes et bordé de portiques qui en facilitent la fréquentation, un «bâtiment commercial» proche de celui qui a été récemment fouillé à Saint-Romain-en-Gal. La fontaine se trouve immédiatement à l'est de cet édifice, mais comme elle s'inscrit dans le système à $160^{\circ}$, elle en est séparée par un espace triangulaire qui assure le raccord entre les deux orientations et offre un dégagement le long du portique oriental.

4 L'organisation urbaine apparaît désormais assez clairement dans ce secteur central où se succèdent, d'ouest en est, trois insulae : l'insula A qui correspond au sanctuaire et dont la bordure nord suit l'orientation initiale entre 0 et $30^{\circ}$ est ; l'insula B qui offre une longueur de quelque $48 \mathrm{~m}$ ( 165 pieds ?) et qui se structure - au moins pour les périodes tardives, seules visibles actuellement - d'après l'orientation à $160^{\circ}$; l'insula $\mathrm{C}$ qui comprend deux édifices relevant d'orientations différentes. Si nous ne savons pas où se trouve la bordure orientale de cette insula, du moins connaissons-nous maintenant le contexte urbanistique dans lequel s'insère la fontaine : située à l'intérieur d'un îlot, elle ouvre ses deux volées de marches sur les rues qui la bordent au nord et au sud, tandis que ses deux longs côtés aveugles- s'inscrivent dans un tissu urbain dont le bâtiment commercial et l'espace triangulaire qui s'installent à l'ouest suggèrent toute la complexité.

5 Cette première campagne de fouille a livré d'autres informations. Nous ne développerons pas cette année celles qui concernent la ville tardive, car si elles apparaissent multiples, elles ont besoin d'être précisées. Nous insisterons en revanche sur la moitié orientale de l'insula B. Il s'agit de la zone qui correspond à une légère dépression sur la carte topographique et à un secteur de faible résistivité sur celle des prospections géophysiques. Le décapage ayant révélé très rapidement la présence d'une vaste fosse ovale, nous avons décidé d'y mener un sondage mécanique, complété par des observations stratigraphiques. Celles-ci ont révélé que la dépression remonte à la période antique, car elle est comblée dans sa partie supérieure par des remblais contenant de la céramique des trois premiers siècles apr. J.-C., mais où ne figure, jusqu'ici, aucun élément caractéristique $\mathrm{du} \mathrm{IV}^{\mathrm{e}} \mathrm{s}$. La question qui se pose est celle de l'origine d'une telle fosse. Tout en envisageant qu'elle ait pu être ouverte pour construire - ou récupérer? - une structure, nous nous demandons également, à la suite de J. Despriée et de R. Gageonnet frappés par le comblement en forme d'entonnoir, s'il ne s'agirait pas d'un fontis réactivé à l'époque antique. On sait en effet que le plateau calcaire qui domine la Creuse est une structure karstique. La présence d'un fontis - comblé à plusieurs reprises et utilisé même, à un moment, comme puits perdu - pourrait expliquer le gel du secteur et la 
transformation de la zone qui l'entoure en un vaste espace non construit, simplement recouvert d'un sol de galets plusieurs fois rechargé. Des carottages seront réalisés en 1998 par le Département Géotechnique du Ministère de l'Equipement à Blois, et s'ils confirment l'hypothèse du fontis, il sera particulièrement intéressant d'observer la façon dont les ingénieurs ou urbanistes gallo-romains ont traité ce phénomène naturel, qu'il se soit révélé de lui-même, ou qu'il ait été provoqué par l'homme.

Enfin, cette fouille devrait permettre de mieux connaître les derniers moments de la ville, de sa destruction, de sa ruine et de son exploitation comme carrière. Elle a en effet fait apparaître des aires de débitage de blocs sculptés, ainsi que des fosses dans lesquelles avaient été jetés de nombreux fragments calcaires. La découverte de quelques tessons de céramique glaçurée verte dans l'une de ces fosses, ainsi que dans la tranchée de récupération d'un des murs du secteur occidental de l'insula $B$, révèle les interventions de l'époque médiévale. Les $\mathrm{XIII}^{\mathrm{e}}, \mathrm{XIV}^{\mathrm{e}}$ et $\mathrm{XV}^{\mathrm{e}} \mathrm{s}$. pourraient ainsi correspondre sur le plateau des Mersans aux derniers moments de la longue période d'abandon et de récupération du site avant sa mise en culture.

\section{INDEX}

Index géographique : Centre, Indre (36), Saint-Marcel

Mots-clés : insula, fontaine, tissu urbain, fontis

Index chronologique : Gallo-romain, Moyen Âge

operation Prospection géophysique (PG) 